HETEROCYCLES, Vol. , No. , , pp. -. (c) The Japan Institute of Heterocyclic Chemistry

Received, , Accepted, , Published online, .COM-06-(Please do not delete.)

\title{
A SIMPLE AND CONVENIENT HIGH YIELDING SYNTHESIS OF SUBSTITUTED ISOINDOLINES
}

\section{Keith Smith,* Gamal A. El-Hiti, Amany S. Hegazy, and Ahmed Fekri}

School of Chemistry, Cardiff University, Main Building, Park Place, Cardiff, CF10 3AT, UK; e-mail: smithk13@cardiff.ac.uk and el-hitiga@cardiff.ac.uk

\begin{abstract}
Trifluoroacetic anhydride-induced dehydration of substituted 2-(pivaloylaminomethyl)phenyl- and 2-(dimethylaminocarbonylmethyl)phenylmethanols gives the corresponding isoindolines in excellent yields when there are aryl substituents at the methanol carbon atom.
\end{abstract}

\section{INTRODUCTION}

Isoindoles represent the core unit of numerous naturally occurring substances. ${ }^{1-7}$ However, isoindoles are commercially less important than indoles, at least partly because of a lack of good methods for their synthesis. Simple isoindoles can be prepared from 1,2-bis(bromomethyl)benzene, 2-cyanobenzyl chloride, phthalaldehyde or other carbonyl compounds. ${ }^{8-14}$ Unfortunately, such methods are less useful for isoindole compounds with substituents on the benzene ring. However, Clayden has developed a very useful approach to dihydroisoindolones with particular substitution patterns using dearomatising cyclization of lithiated $N$-benzylbenzamides and demonstrated its utility for synthesis of natural products such as kainic acid. ${ }^{15-19}$ We have also been interested in efficient lithiation procedures for the production of substituted aromatics and heteroaromatics that might be difficult to prepare by other means. ${ }^{20}$ We have recently developed convenient procedures for the directed lithiation of compounds of the general formula 1 (Figure 1) using $t$-BuLi in anhydrous THF at $-78{ }^{\circ} \mathrm{C}$, followed by reactions with carbonyl compounds (aldehydes and ketones) to give the corresponding ortho-disubstituted products 2 in high yields. ${ }^{21}$ In principle, compounds 2 could be cyclized to the corresponding isoindolines $\mathbf{3}$. We therefore decided to investigate this possibility using trifluoroacetic anhydride (TFAA) as the dehydrating agent and now report success in obtaining the corresponding isoindolines 3 in excellent yields when at least one of the groups $\mathrm{R}^{3}$ or $\mathrm{R}^{4}$ is an aryl group.

Dedicated to Professor Akira Suzuki on the occasion of his $80^{\text {th }}$ birthday. 
<smiles>[R]C(=O)NCc1ccc([R])cc1</smiles>

1<smiles>[R]C(=O)NCc1ccc([R])cc1C([R])([R])O</smiles>

2<smiles>[R]C(=O)N1Cc2ccc([R])cc2C1([R])[R]</smiles>

3

$\mathrm{R}^{1}=\mathrm{H}, \mathrm{OMe}, \mathrm{Me}, \mathrm{Cl}, \mathrm{F} ; \mathrm{R}^{2}={ }^{t} \mathrm{Bu}, \mathrm{NMe}_{2} ; \mathrm{R}^{3}, \mathrm{R}^{4}=\mathrm{H}, \mathrm{Ar},\left(\mathrm{CH}_{2}\right)_{5}$

Figure 1. Structures of compounds 1-3.

\section{RESULTS AND DISCUSSION}

An initial reaction was carried out between compound $\mathbf{4}$ and few drops of TFAA at room temperature in dichloromethane (DCM, Scheme 1). TLC was used to monitor the progress of the reaction and indicated the formation of a new product, the formation of which was complete within 5 minutes. The mixture was quenched by the addition of water and worked-up. The residue obtained was subjected to flash column chromatography (silica gel; $\mathrm{Et}_{2} \mathrm{O}-$ hexane, $1: 3$ ) to give the pure product, identified as compound $\mathbf{5}$, as a white solid in $85 \%$ yield. The structure of $\mathbf{5}$ was confirmed by X-ray crystallography (Figure 2).<smiles></smiles>

4

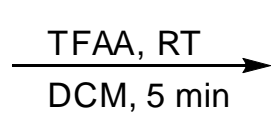

$(85 \%)$

Scheme 1. Synthesis of 5 via dehydration of $\mathbf{4}$ with TFAA.

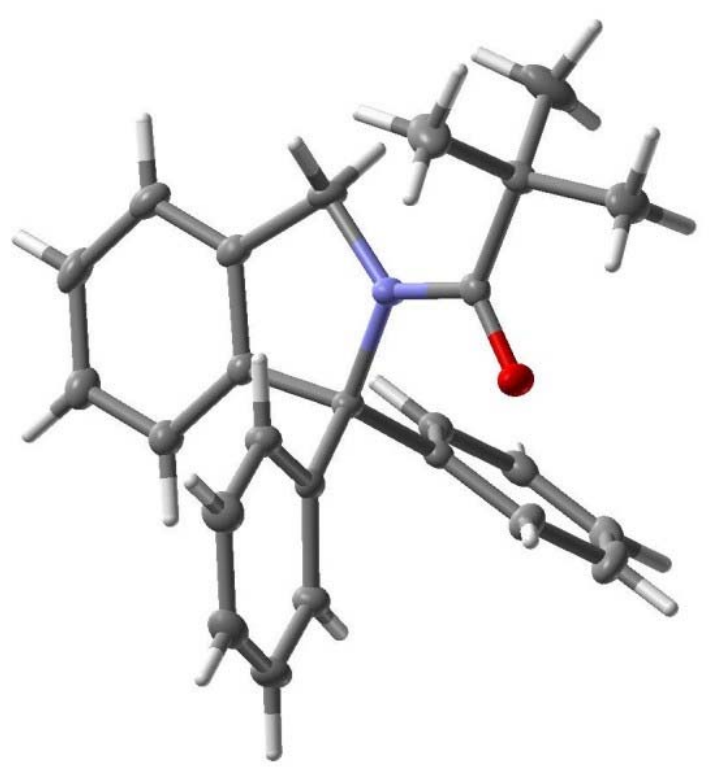

Figure 2. X-Ray crystal structure of compound 5 
A possible mechanism for the formation of $\mathbf{5}$ is shown in Scheme 2.<smiles>O=C(Cc1ccccc1)NCc1ccccc1C(OC(=O)C(F)(F)F)(c1ccccc1)c1ccccc1CNC(=O)Cc1ccccc1C(=Cc1ccccc1)c1ccccc1</smiles>

4<smiles>C[C+](C)CC(=O)[N+]1(c2ccccc2)Cc2ccccc2C1(c1ccccc1)c1ccccc1</smiles>

5

Scheme 2. A possible mechanism for the dehydration of $\mathbf{4}$ on treatment with TFAA

Having found that reaction of compound $\mathbf{4}$ with TFAA occurred smoothly and rapidly, it was of interest to see if the cyclization reaction of other compounds related to 4 would be useful and general. Consequently, reactions of various compounds of the type $\mathbf{2}$ with TFAA were carried out at room temperature in DCM for 5 minutes (Scheme 3). Each reaction was conducted under identical conditions and the crude products were subjected to flash column chromatography to give the corresponding isoindolines 6-24 (Scheme 3) in excellent yields (Table 1).<smiles>[R]C(=O)NCc1ccc([R])cc1C([R])([R])O</smiles>

2

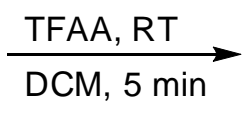

6-24

Scheme 3. Synthesis of various substituted isoindolines 6-24

The ${ }^{1} \mathrm{H}$ NMR spectra of compounds where $\mathrm{R}^{3}$ and $\mathrm{R}^{4}$ are different (i.e. compounds 6-8, 10, 11, 13, 15, 17, 19 and 21-24) showed diastereotopicity for the two hydrogens of the $\mathrm{CH}_{2}$ group at position 3 of the isoindoline ring. The two-dimensional NMR correlation spectroscopy (COSY) for compound 17 showed that one of the hydrogens at the 3-position was also coupled to H-1. The structures of compounds 8 (Figure 3) and 17 (Figure 4) were confirmed by X-ray crystallography. 
Table 1: Synthesis of isoindolines 5-24 via dehydration of $\mathbf{2}$ according to Schemes 1 and 3

\begin{tabular}{llllll}
\hline Product & $\mathrm{R}^{1}$ & $\mathrm{R}^{2}$ & $\mathrm{R}^{3}$ & $\mathrm{R}^{4}$ & Yield (\%) $^{a}$ \\
\hline $\mathbf{5}$ & $\mathrm{H}$ & ${ }^{t} \mathrm{Bu}$ & $\mathrm{Ph}$ & $\mathrm{Ph}$ & 85 \\
$\mathbf{6}$ & $\mathrm{H}$ & ${ }^{t} \mathrm{Bu}$ & $\mathrm{H}$ & $4-\mathrm{MeOC}_{6} \mathrm{H}_{4}$ & 89 \\
$\mathbf{7}$ & $\mathrm{H}$ & ${ }^{t} \mathrm{Bu}$ & $\mathrm{H}$ & $\mathrm{Ph}$ & 87 \\
$\mathbf{8}$ & $\mathrm{OMe}$ & ${ }^{t} \mathrm{Bu}$ & $\mathrm{H}$ & $4-\mathrm{MeOC}_{6} \mathrm{H}_{4}$ & 90 \\
$\mathbf{9}$ & $\mathrm{OMe}$ & ${ }^{t} \mathrm{Bu}$ & $\mathrm{Ph}$ & $\mathrm{Ph}$ & 98 \\
$\mathbf{1 0}$ & $\mathrm{Me}$ & ${ }^{t} \mathrm{Bu}$ & $\mathrm{H}$ & $4-\mathrm{MeOC}_{6} \mathrm{H}_{4}$ & 88 \\
$\mathbf{1 1}$ & $\mathrm{Cl}$ & ${ }^{t} \mathrm{Bu}$ & $\mathrm{H}$ & $4-\mathrm{MeOC}_{6} \mathrm{H}_{4}$ & 88 \\
$\mathbf{1 2}$ & $\mathrm{Cl}$ & ${ }^{t} \mathrm{Bu}$ & $\mathrm{Ph}$ & $\mathrm{Ph}$ & 77 \\
$\mathbf{1 3}$ & $\mathrm{F}$ & ${ }^{t} \mathrm{Bu}$ & $\mathrm{H}$ & $4-\mathrm{MeOC}_{6} \mathrm{H}_{4}$ & 84 \\
$\mathbf{1 4}$ & $\mathrm{F}$ & ${ }^{t} \mathrm{Bu}_{\mathbf{1 0}}$ & $\mathrm{Ph}$ & $\mathrm{Ph}$ & 84 \\
$\mathbf{1 5}$ & $\mathrm{H}$ & $\mathrm{NMe} 2$ & $4-\mathrm{MeOC}_{6} \mathrm{H}_{4}$ & 89 \\
$\mathbf{1 6}$ & $\mathrm{NMe} 2$ & $\mathrm{Ph}$ & $\mathrm{Ph}$ & 91 \\
$\mathbf{1 7}$ & $\mathrm{H}$ & $\mathrm{H}$ & $4-\mathrm{MeOC}_{6} \mathrm{H}_{4}$ & 91 \\
$\mathbf{1 8}$ & $\mathrm{OMe}$ & $\mathrm{NMe}_{2}$ & $\mathrm{Ph}$ & $\mathrm{Ph}$ & 88 \\
$\mathbf{1 9}$ & $\mathrm{OMe}$ & $\mathrm{NMe}_{2}$ & $\mathrm{H}$ & $4-\mathrm{MeOC}_{6} \mathrm{H}_{4}$ & 91 \\
$\mathbf{2 0}$ & $\mathrm{Me}$ & $\mathrm{NMe}_{2}$ & $\mathrm{Ph}$ & $\mathrm{Ph}$ & 88 \\
$\mathbf{2 1}$ & $\mathrm{Me}$ & $\mathrm{NMe}_{2}$ & $\mathrm{H}$ & $4-\mathrm{MeOC}_{6} \mathrm{H}_{4}$ & 78 \\
$\mathbf{2 2}$ & $\mathrm{Cl}$ & $\mathrm{NMe}_{2}$ & $\mathrm{H}$ & 4 & 78 \\
$\mathbf{2 3}$ & $\mathrm{Cl}$ & $\mathrm{NMe}_{2}$ & $\mathrm{H}$ & $4-\mathrm{MeOC}_{6} \mathrm{H}_{4}$ & 85 \\
$\mathbf{2 4}$ & $\mathrm{F}$ & $\mathrm{NMe}_{2}$ & $\mathrm{H}$ & $\mathrm{Ph}$ & 85 \\
\hline
\end{tabular}

${ }^{a}$ Yield of isolated product after purification by column chromatography.

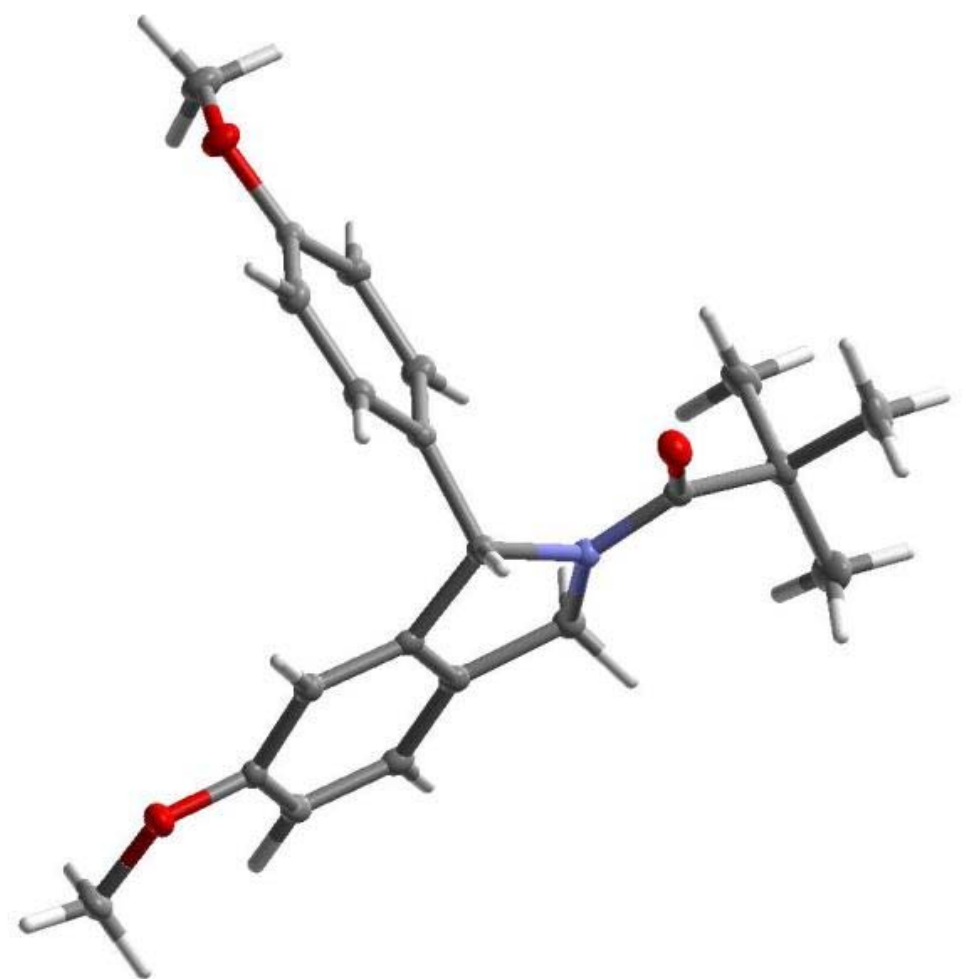

Figure 3. X-Ray crystal structure of compound 8 


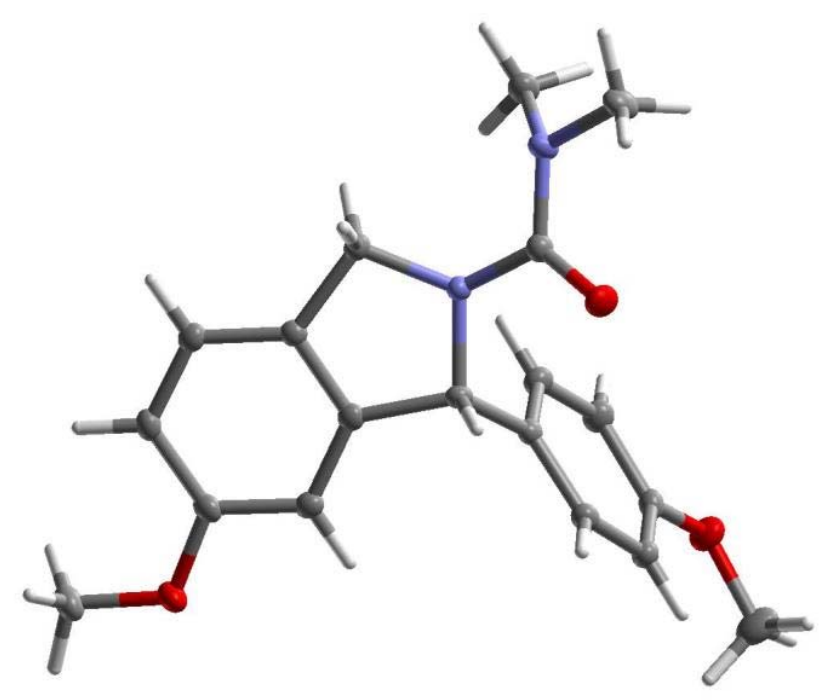

Figure 4. X-Ray crystal structure of compound 17

From the results in Table 1 it is clear that the cyclization is quite a general process, producing various substituted isoindolines in excellent yields regardless of the nature of the ring substituent $\mathrm{R}^{1}$, of whether $\mathrm{R}^{2}$ was $\mathrm{Bu}^{t}$ or $\mathrm{NMe}_{2}$, or whether the groups at position 1 were two phenyl groups or one hydrogen and one aryl group. The combination of directed lithiation of $N$-pivaloyl- or $N$-dimethylaminocarbonyl-benzylamines, addition of an aromatic aldehyde or ketone, ${ }^{21}$ and then treatment with TFAA therefore represents a very simple and direct approach to substituted isoindolines.

We next attempted to extend the process to compounds of type 2 in which the group $C(O H) R^{3} R^{4}$ had been derived from cyclohexanone. It was found, however, that reactions of $N$-(2-(1-hydroxycyclohexyl)benzyl)pivalamide (25) and $N$-(2-(1-hydroxycyclohexyl)-4-methoxybenzyl)pivalamide (26) with TFAA in DCM at room temperature proceeded in a different manner (Scheme 4). The crude products were purified by column chromatography to give $\mathbf{2 7}$ and 28, respectively, in high yields (Scheme 4).
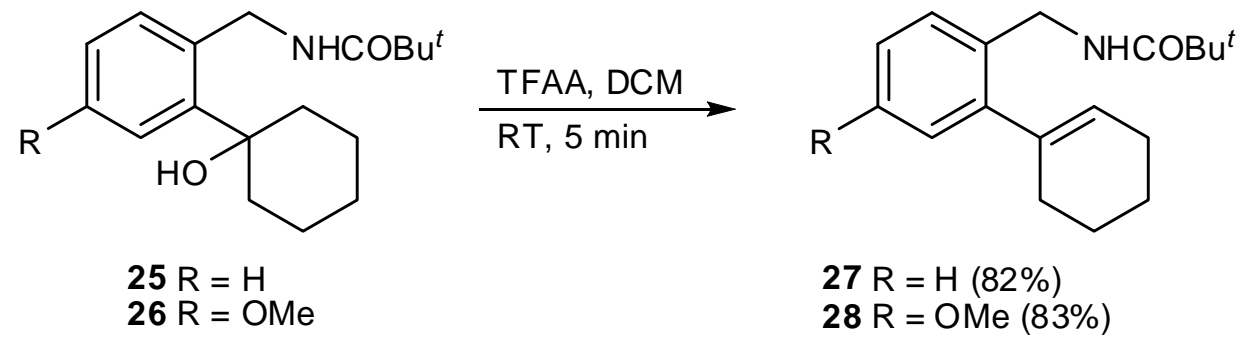

Scheme 4. Synthesis of compounds 27 and 28

The reaction presumably proceeds via a cation similar to that shown in Scheme 2, but loss of a proton from the adjacent position, impossible when the attached groups are aromatic, is easier, leading to an E1 elimination process. 
We attempted reactions of $\mathbf{2 5}$ and $\mathbf{2 6}$ with TFAA at room temperature for longer reaction times (up to $24 \mathrm{~h}$ ) but products $\mathbf{2 7}$ and $\mathbf{2 8}$ were obtained, respectively, in yields comparable to those obtained from the reactions shown in Scheme 4. No further attempts were made to try to find conditions under which cyclization of $\mathbf{2 5}$ and $\mathbf{2 6}$ to the corresponding substituted isoindolines could be effective.

\section{CONCLUSION}

Dehydration of various substituted benzylamines with trifluoroacetic anhydride gave the corresponding isoindolines in excellent yields. The process is general, simple and convenient for compounds having aromatic groups attached to the carbinol carbon atom. However, when a hydroxycyclohexyl group is present at the 2-position, dehydration produces cyclohexenyl derivatives in high yield, rather than giving isoindoline derivatives.

\section{EXPERIMENTAL}

GENERAL METHODS: Melting point determinations were performed by the open capillary method using a Gallenkamp melting point apparatus and are reported uncorrected. ${ }^{1} \mathrm{H}$ and ${ }^{13} \mathrm{C}$ NMR spectra were recorded on a Bruker AV400 or AV500 spectrometer operating at 400 or $500 \mathrm{MHz}$ for ${ }^{1} \mathrm{H}$ and 100 or 125 $\mathrm{MHz}$ for ${ }^{13} \mathrm{C}$ measurements, respectively. Chemical shifts $\delta$ are reported in parts per million (ppm) relative to TMS and coupling constants $J$ are in $\mathrm{Hz}$ and have been rounded to the nearest whole number. ${ }^{13} \mathrm{C}$ multiplicities are based on DEPT signals. Assignments of signals are based on coupling patterns and expected chemical shift values and have not been rigorously confirmed. Signals with similar characteristics might be interchanged. Low-resolution mass spectra were recorded on a Quattro II spectrometer, electron impact (EI) at $70 \mathrm{eV}$ and chemical ionization (CI) at $50 \mathrm{eV}$ by the use of $\mathrm{NH}_{3}$ as ionization gas. Accurate mass data were obtained on a MAT900 instrument. Electrospray (ES) analyses were performed on a ZQ4000 spectrometer in positive and negative ionisation modes. IR spectra were recorded on a Perkin Elmer Spectrum One FT-IR spectrometer or a Perkin Elmer 1600 series FT-IR Spectrometer. Microanalyses were performed by Warwick analytical service at the University of Warwick. Column chromatography was carried out using Fischer Scientific silica 60A (35-70 micron). Alkyllithiums were obtained from Aldrich Chemical Company and were estimated prior to use by the method of Watson and Eastham. ${ }^{22}$ Other chemicals were obtained from Aldrich Chemical Company and used without further purification. THF was distilled from sodium benzophenone ketyl. Other solvents were purified by standard procedures. $^{23,24}$

Substituted aryl methanols 2: A solution of $t$-BuLi in heptane (2.6 mL, 1.7 M, $4.4 \mathrm{mmol})$ was added to a cold $\left(-78{ }^{\circ} \mathrm{C}\right)$, stirred solution of $\mathbf{1}(2.0 \mathrm{mmol})$ in anhydrous THF $(20 \mathrm{~mL})$ under $\mathrm{N}_{2}$. The mixture was stirred at $-78{ }^{\circ} \mathrm{C}$ for $4 \mathrm{~h}$, after which an aldehyde or ketone $(2.2 \mathrm{mmol})$, in anhydrous THF (8 $\left.\mathrm{mL}\right)$ if solid, 
otherwise neat, was added. The mixture was stirred for $2 \mathrm{~h}$ at $-78^{\circ} \mathrm{C}$ then the cooling bath was removed and the mixture allowed to warm to room temperature. It was then diluted with $\mathrm{Et}_{2} \mathrm{O}(10 \mathrm{~mL})$ and quenched with aq. sat. $\mathrm{NH}_{4} \mathrm{Cl}(10 \mathrm{~mL})$. The organic layer was separated, washed with $\mathrm{H}_{2} \mathrm{O}(2 \times 10 \mathrm{~mL})$, dried $\left(\mathrm{MgSO}_{4}\right)$, and evaporated under reduced pressure. The residue obtained was purified by column chromatography (silica gel; $\mathrm{Et}_{2} \mathrm{O}$-hexane, 1:3) to give the corresponding product $2 .^{21}$

1,1-Diphenyl-2-pivaloylisoindoline (5): Trifluoroacetic anhydride $(0.5 \mathrm{~mL})$ was added to a stirred solution of $\mathrm{N}$-(2-(hydroxydiphenylmethyl)benzyl)pivalamide (4; $0.50 \mathrm{~g}, 1.34 \mathrm{mmol})$ in DCM (10 mL) at room temperature. The mixture was stirred for $5 \mathrm{~min}$ at room temperature, after which TLC showed the formation of a single product. The reaction mixture was quenched with $\mathrm{H}_{2} \mathrm{O}(10 \mathrm{~mL})$. The organic layer was separated, washed with aq. sat. $\mathrm{NaHCO}_{3}(10 \mathrm{~mL}), \mathrm{H}_{2} \mathrm{O}(2 \times 10 \mathrm{~mL})$, dried $\left(\mathrm{MgSO}_{4}\right)$, and evaporated under reduced pressure. The residue obtained was subjected to flash column chromatography (silica gel; $\mathrm{Et}_{2} \mathrm{O}$-hexane, 1:3) to give pure 5 (0.40 g, $\left.1.14 \mathrm{mmol}, 85 \%\right)$ as a white solid. Mp $150{ }^{\circ} \mathrm{C}$. ${ }^{1} \mathrm{H}$ NMR (400 MHz, $\mathrm{CDCl}_{3}$ ): $\delta=7.41-7.23$ (m, 13 H, 2 Ph, H-4, H-5 and H-6), 7.04 (d, J= 8 Hz, 1 H, H-7), 5.30 (s, 2 H, H-3), 1.35 [s, $9 \mathrm{H}, \mathrm{C}\left(\mathrm{CH}_{3}\right)_{3}$ ]. ${ }^{13} \mathrm{C}$ NMR (100 MHz, $\left.\mathrm{CDCl}_{3}\right): \delta=174.8$ (s, C=O), 146.5 (s, C-7a), 143.1 (s, C-1 of 2 Ph), 135.7 (s, C-3a), 128.8 (d, C-2/C-6 of 2 Ph), 128.5 (d, C-4), 128.0 (d, C-7), 127.9 (d, C-3/C-5 of $2 \mathrm{Ph}$ ), 127.0 (d, C-4 of 2 Ph), 125.1 (d, C-6), 122.2 (d, C-5), 80.9 (s, C-1), 53.9 (t, C-3), 40.1 [s, C( $\left.\mathrm{CH}_{3}\right)_{3}$ ],

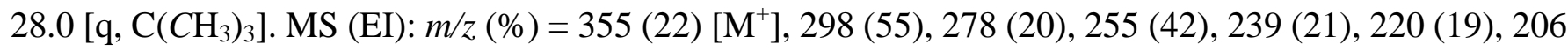
(12), 194 (40), 178 (28), 165 (32), 116 (11), 105 (23), 91 (9), 77 (20), 57 (100), 41 (27). MS (CI): m/z (\%) = 356 (100) $[\mathrm{MH}]^{+}$. HRMS (CI): $\mathrm{m} / \mathrm{z}$ calcd. for $\mathrm{C}_{25} \mathrm{H}_{26} \mathrm{NO}[\mathrm{MH}]^{+}$356.2009; found 356.2010. IR (FT): $v=$ 2957, 1618, 1510, 1371, 1348, 1242, 1173, $1030 \mathrm{~cm}^{-1}$. Anal. Calcd for $\mathrm{C}_{25} \mathrm{H}_{25} \mathrm{NO}$ (355.194): C 84.47, H 7.09, N, 3.94. Found: C 84.35, H 7.03, N 3.83\%.

Substituted isoindolines 6-24: The procedure was identical to that described for the synthesis of 5 except that compounds $2(0.50 \mathrm{~g})$ were used as starting materials instead of $\mathbf{4}$. The residues obtained after work-up were subjected to flash column chromatography (silica gel; $\mathrm{Et}_{2} \mathrm{O}$-hexane, 1:3) to give the pure products 6-24 as white solids.

1-(4-Methoxyphenyl)-2-pivaloylisoindoline (6): 0.42 g (89\%). Mp 112-114 ${ }^{\circ} \mathrm{C} .{ }^{1} \mathrm{H}$ NMR (400 MHz, $\mathrm{CDCl}_{3}$ ): $\delta=7.32$ (app. t, $J=8 \mathrm{~Hz}, 1 \mathrm{H}, \mathrm{H}-6$ ), 7.29 (d, $J=8 \mathrm{~Hz}, 1 \mathrm{H}, \mathrm{H}-4$ ), 7.25 (app. t, $J=8 \mathrm{~Hz}, 1 \mathrm{H}, \mathrm{H}-5$ ), 7.19 (d, $J=9 \mathrm{~Hz}, 2 \mathrm{H}, \mathrm{H}-2 / \mathrm{H}-6$ of 4-methoxyphenyl), 7.09 (d, $J=8 \mathrm{~Hz}, 1 \mathrm{H}, \mathrm{H}-7$ ), 6.83 (d, $J=9 \mathrm{~Hz}, 2 \mathrm{H}$, H-3/H-5 of 4-methoxyphenyl), 6.32 (s, 1 H, H-1), 5.26-5.16 (m, 2 H, H-3a and H-3b), 3.78 (s, 3 H, OCH ), $^{-}$ $1.24\left[\mathrm{~s}, 9 \mathrm{H}, \mathrm{C}\left(\mathrm{CH}_{3}\right)_{3}\right] .{ }^{13} \mathrm{C} \mathrm{NMR}\left(100 \mathrm{MHz}, \mathrm{CDCl}_{3}\right): \delta=176.8$ (s, C=O), 159.0 (s, C-4 of 4-methoxyphenyl), 141.4 (s, C-7a), 136.3 (s, C-3a), 128.3 (d, C-4 and C-7), 128.1 (s, C-1 of 4-methoxyphenyl), 128.0 (d, C-2/C-6 of 4-methoxyphenyl), 123.9 (d, C-6), 122.7 (d, C-5), 114.3 (d, 
C-3/C-5 of 4-methoxyphenyl), 69.0 (d, C-1), 55.6 (q, $\mathrm{OCH}_{3}$ ), 54.0 (t, C-3), 39.7 [s, $\mathrm{C}\left(\mathrm{CH}_{3}\right)_{3}$ ], 28.0 [q, $\left.\mathrm{C}\left(\mathrm{CH}_{3}\right)_{3}\right]$. MS (EI): $\mathrm{m} / \mathrm{z}(\%)=309$ (12) [M] ${ }^{+}, 294$ (3), 278 (7), 252 (15), 224 (12), 209 (23), 194 (8), 165 (9), 135 (7), 116 (6), 57 (100), 41 (73). MS (CI): m/z (\%) = 310 (100) [MH] ${ }^{+}$. HRMS (CI): m/z calcd. for $\mathrm{C}_{20} \mathrm{H}_{24} \mathrm{NO}_{2}[\mathrm{MH}]^{+}$310.1802; found 310.1802. IR (FT): $v=2958,1616,1510,1371,1357,1242,1117 \mathrm{~cm}^{-1}$. Anal. Calcd for $\mathrm{C}_{20} \mathrm{H}_{23} \mathrm{NO}_{2}$ (309.173): C 77.64, H 7.49, N 4.53. Found: C 77.65, H 7.54, N 4.52\%.

1-Phenyl-2-pivaloylisoindoline (7): $0.41 \mathrm{~g}$ (87\%). Mp 110-111 ${ }^{\circ} \mathrm{C} .{ }^{1} \mathrm{H}$ NMR $\left(400 \mathrm{MHz}, \mathrm{CDCl}_{3}\right): \delta=$ 7.23-7.11 (m, 8 H, Ph, H-4, H-5 and H-6), 7.00 (d, $J=8$ Hz, 1 H, H-7), 6.24 (s, 1 H, H-1), 5.17-5.07 (m, 2 H, H-3a and H-3b), 1.24 [s, $\left.9 \mathrm{H}, \mathrm{C}\left(\mathrm{CH}_{3}\right)_{3}\right] .{ }^{13} \mathrm{C}$ NMR (100 MHz, $\left.\mathrm{CDCl}_{3}\right): \delta=175.2$ (s, C=O), 143.9 (s, C-1 of Ph), 141.0 (s, C-7a), 136.2 (s, C-3a), 129.0 (d, C-3/C-5 of Ph), 128.4 (d, C-4), 128.1 (d, C-2/C-6 of Ph), 127.6 (d, C-4 of Ph), 126.7 (d, C-7), 123.9 (d, C-6), 122.7 (d, C-5), 69.7 (d, C-1), 54.2 (t, C-3), 39.8 [s, $C\left(\mathrm{CH}_{3}\right)_{3}$ ], 28.0 [q, $\left.\mathrm{C}\left(\mathrm{CH}_{3}\right)_{3}\right]$. MS (EI): $\mathrm{m} / \mathrm{z}(\%)=279$ (11) [M] , 222 (10), 194 (11), 179 (32), 178 (29), 165 (16), 116 (12), 105 (6), 91 (9), 89 (15), 77 (18), 57 (100), 41 (95). MS (CI): m/z (\%) = 297 (2) [M + NH $\left.]_{4}\right]^{+}$, 280 (100) [MH] ${ }^{+}$. HRMS (CI): $\mathrm{m} / \mathrm{z}$ calcd. for $\mathrm{C}_{19} \mathrm{H}_{22} \mathrm{NO}[\mathrm{MH}]^{+}$280.1696; found 280.1694. IR (FT): $v=$ 2962, 1616, 1510, 1410, 1369, 1357, 1209, $1093 \mathrm{~cm}^{-1}$.

6-Methoxy-1-(4-methoxyphenyl)-2-pivaloylisoindoline (8): 0.43 g (90\%). Mp 133-134 ${ }^{\circ} \mathrm{C} .{ }^{1} \mathrm{H}$ NMR (400 MHz, $\mathrm{CDCl}_{3}$ ): $\delta=7.21$ (d, $J=8 \mathrm{~Hz}, 1 \mathrm{H}, \mathrm{H}-4$ ), 7.18 (d, $J=9 \mathrm{~Hz}, 2 \mathrm{H}, \mathrm{H}-2 / \mathrm{H}-6$ of 4-methoxyphenyl), 6.85 (dd, $J=2,8 \mathrm{~Hz}, 1 \mathrm{H}, \mathrm{H}-5$ ), 6.83 (d, $J=9 \mathrm{~Hz}, 2 \mathrm{H}, \mathrm{H}-3 / \mathrm{H}-5$ of 4-methoxyphenyl), 6.58 (d, $J=2 \mathrm{~Hz}, 1$

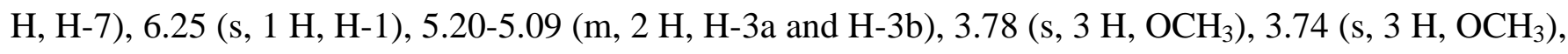
1.32 [s, $\left.9 \mathrm{H}, \mathrm{C}\left(\mathrm{CH}_{3}\right)_{3}\right] .{ }^{13} \mathrm{C}$ NMR $\left(100 \mathrm{MHz}, \mathrm{CDCl}_{3}\right): \delta=176.7$ (s, C=O), 160.2 (s, C-6), 159.0 (s, C-4 of 4-methoxyphenyl), 142.8 (s, C-7a), 136.2 (s, C-1 of 4-methoxyphenyl and C-3a), 128.2 (d, C-4), 123.5 (d, C-7), 114.9 (d, C-5), 114.3 (d, C-2/C-6 of 4-methoxyphenyl), 108.5 (d, C-3/C-5 of 4-methoxyphenyl), 69.1 (d, C-1), 55.9 (q, $\mathrm{OCH}_{3}$ ), 55.6 (q, $\left.\mathrm{OCH}_{3}\right), 53.5$ (t, C-3), 39.7 [s, $\left.\mathrm{C}\left(\mathrm{CH}_{3}\right)_{3}\right], 28.0$ [q, $\mathrm{C}\left(\mathrm{CH}_{3}\right)_{3}$ ]. MS (EI): m/z (\%) = 339 (8) [M] , 282 (41), 254 (12), 239 (16), 238 (20), 224 (7), 208 (6), 195 (5), 165 (8), 152 (8), 139 (7), 104 (8), 92 (5), 77 (14), 57 (100), 41 (73). MS (CI): m/z (\%) = 340 (100) [MH] ${ }^{+}$. HRMS (CI): m/z calcd. for $\mathrm{C}_{21} \mathrm{H}_{26} \mathrm{NO}_{3}[\mathrm{MH}]^{+}$340.1907; found 340.1913. IR (FT): $v=2959,1615,1511,1477,1400,1358,1243$, 1171, $1023 \mathrm{~cm}^{-1}$. Anal. Calcd for $\mathrm{C}_{21} \mathrm{H}_{25} \mathrm{NO}_{3}$ (339.183): C 74.31, H 7.42, N 4.13. Found: C 74.42, H 7.46, N $4.08 \%$.

6-Methoxy-1,1-diphenyl-2-pivaloylisoindoline (9): $0.47 \mathrm{~g}$ (98\%). Mp 119-121 ${ }^{\circ} \mathrm{C} .{ }^{1} \mathrm{H}$ NMR (400 MHz, $\mathrm{CDCl}_{3}$ ): $\delta=7.26-7.10$ (m, $11 \mathrm{H}, 2 \mathrm{Ph}$ and H-4), 6.73 (dd, $\left.J=2,8 \mathrm{~Hz}, 1 \mathrm{H}, \mathrm{H}-5\right), 6.37$ (d, $J=2 \mathrm{~Hz}, 1 \mathrm{H}$, $\mathrm{H}-7$ ), 5.11 (s, $2 \mathrm{H}, \mathrm{H}-3$ ), 3.56 (s, $\left.3 \mathrm{H}, \mathrm{OCH}_{3}\right), 1.20$ [s, $\left.9 \mathrm{H}, \mathrm{C}\left(\mathrm{CH}_{3}\right)_{3}\right] .{ }^{13} \mathrm{C} \mathrm{NMR}\left(100 \mathrm{MHz}, \mathrm{CDCl}_{3}\right): \delta=$ 175.2 (s, C=O), 160.2 (s, C-6), 147.6 (s, C-7a), 142.7 (s, C-1 of 2 Ph), 128.7 (d, C-3/C-5 of 2 Ph), 128.4 (s, C-3a), 127.9 (d, C-2/C-6 of 2 Ph), 127.15 (d, C-4 of 2 Ph), 123.0 (d, C-4), 114.8 (d, C-7), 110.0 (d, C-5), 
81.2 (s, C-1), 55.8 (q, $\left.\mathrm{OCH}_{3}\right), 53.5$ (t, C-3), 40.2 [s, $\left.\mathrm{C}\left(\mathrm{CH}_{3}\right)_{3}\right], 27.88$ [q, $\left.\mathrm{C}\left(\mathrm{CH}_{3}\right)_{3}\right] . \mathrm{MS}(\mathrm{EI}): \mathrm{m} / \mathrm{z}(\%)=386$ (22) $[\mathrm{M}+1]^{+}, 385$ (81) [M] $]^{+}, 328$ (100), 308 (42), 300 (38), 292 (21), 220 (16). MS (CI): m/z (\%) = 386 (100) $[\mathrm{MH}]^{+}, 328$ (3), 303 (19), 284 (5). HRMS (CI): m/z calcd. for $\mathrm{C}_{26} \mathrm{H}_{28} \mathrm{NO}_{2}[\mathrm{MH}]^{+}$386.2115; found 386.2113. IR (FT): $v=2957,1638,1599,1490,1444,1351,1210,1172 \mathrm{~cm}^{-1}$.

1-(4-Methoxyphenyl)-6-methyl-2-pivaloylisoindoline (10): $0.42 \mathrm{~g}$ (88\%). Mp 148-149 ${ }^{\circ} \mathrm{C} .{ }^{1} \mathrm{H}$ NMR (400 MHz, $\mathrm{CDCl}_{3}$ ): $\delta=7.22$ (d, $J=8 \mathrm{~Hz}, 1 \mathrm{H}, \mathrm{H}-4$ ), $7.18 \mathrm{~d}, J=9 \mathrm{~Hz}, 2 \mathrm{H}, \mathrm{H}-2 / \mathrm{H}-6$ of 4-methoxyphenyl), 7.10 (d, $J=8$ Hz, 1 H, H-5), 6.90 (s, 1 H, H-7), 6.84 (d, $J=9$ Hz, 2 H, H-3/H-5 of 4-methoxyphenyl), 6.26 (s, $1 \mathrm{H}, \mathrm{H}-1$ ), 5.21-5.11 (m, $2 \mathrm{H}, \mathrm{H}-3 \mathrm{a}$ and H-3b), 3.78 (s, $3 \mathrm{H}, \mathrm{OCH}_{3}$ ), 2.30 (s, $3 \mathrm{H}, \mathrm{CH}_{3}$ ), 1.33 [s, $9 \mathrm{H}$, $\left.\mathrm{C}\left(\mathrm{CH}_{3}\right)_{3}\right] .{ }^{13} \mathrm{C}$ NMR (100 MHz, $\left.\mathrm{CDCl}_{3}\right): \delta=176.7$ (s, C=O), 159.0 (s, C-4 of 4-methoxyphenyl), 141.5 (s, C-7a), 138.1 (s, C-1 of 4-methoxyphenyl), 136.5 (s, C-6), 133.4 (s, C-3a), 128.9 (d, C-2/C-6 of 4-methoxyphenyl), 128.1 (d, C-7), 124.3 (d, C-4), 122.4 (d, C-5), 114.3 (d, C-3/C-5 of 4-methoxyphenyl), 70.0 (d, C-1), 55.6 (q, $\mathrm{OCH}_{3}$ ), 53.8 (t, C-3), 39.7 [s, $\mathrm{C}\left(\mathrm{CH}_{3}\right)_{3}$ ], 28.0 [q, C( $\left.\mathrm{CH}_{3}\right)_{3}$ ], 21.7 (q, $\left.\mathrm{CH}_{3}\right) . \mathrm{MS}(\mathrm{EI})$ : m/z (\%) = 323 (4) [M] $]^{+}, 266$ (3), 238 (8), 223 (13), 208 (9), 194 (6), 165 (8), 130 (7), 77 (14), 57 (100), 41 (77). MS (CI): $m / z(\%)=324$ (100) $[\mathrm{MH}]^{+}, 266$ (3), 216 (5). HRMS (CI): $m / z$ calcd. for $\mathrm{C}_{21} \mathrm{H}_{26} \mathrm{NO}_{2}[\mathrm{MH}]^{+}$ 324.1958; found 324.1953. IR (FT): $v=2958,1637,1535,1357,1231 \mathrm{~cm}^{-1}$. Anal. Calcd for $\mathrm{C}_{21} \mathrm{H}_{25} \mathrm{NO}_{2}$ (323.189): C 77.98, H 7.79, N 4.33. Found: C 77.84, H 7.83, N, 4.41\%.

6-Chloro-1-(4-methoxyphenyl)-2-pivaloylisoindoline (11): 0.42 g (88\%). Mp $137-139{ }^{\circ} \mathrm{C} .{ }^{1} \mathrm{H}$ NMR (500 MHz, $\mathrm{CDCl}_{3}$ ): $\delta=7.25$ (s, $\left.1 \mathrm{H}, \mathrm{H}-7\right), 7.20$ (d, $J=8 \mathrm{~Hz}, 1 \mathrm{H}, \mathrm{H}-5$ ), 7.10 (d, $J=9 \mathrm{~Hz}, 2 \mathrm{H}, \mathrm{H}-2 / \mathrm{H}-6$ of 4-methoxyphenyl), 7.0 (d, $J=8 \mathrm{~Hz}, 1 \mathrm{H}, \mathrm{H}-4), 6.75$ (d, $J=9 \mathrm{~Hz}, 2 \mathrm{H}, \mathrm{H}-3 / \mathrm{H}-5$ of 4-methoxyphenyl),

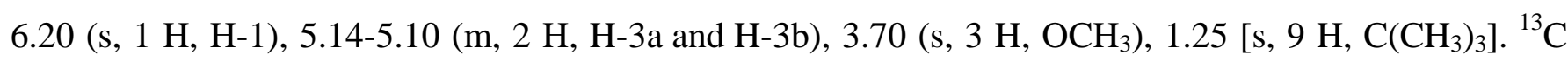
NMR (125 MHz, $\mathrm{CDCl}_{3}$ ): $\delta=178.4$ (s, C=O), 162.7 (s, C-4 of 4-methoxyphenyl), 159.0 (s, C-7a), 142.1 (s, C-3a), 134.1 (s, C-1 of 4-methoxyphenyl), 133.7 (s, C-6), 128.2 (d, C-2/C-6 of 4-methoxyphenyl), 127.9 (d, C-4), 123.8 (d, C-7), 123.5 (d, C-5), 114.1 (d, C-3/C-5 of 4-methoxyphenyl), 68.9 (d, C-1), 55.2 (q, $\left.\mathrm{OCH}_{3}\right), 53.3$ (t, C-3), 39.5 [s, $\left.\mathrm{C}\left(\mathrm{CH}_{3}\right)_{3}\right], 27.3$ [q, $\left.\mathrm{C}\left(\mathrm{CH}_{3}\right)_{3}\right] . \mathrm{MS}(\mathrm{EI}): \mathrm{m} / \mathrm{z}(\%)=345(22)\left[\mathrm{M}^{37} \mathrm{Cl}\right]^{+}$, 343 (67) [M $\left.{ }^{35} \mathrm{Cl}\right]^{+}, 312$ (28), 286 (100), 258 (29), 243 (45), 208 (50), 165 (18), 116 (16), 57 (40). HRMS (EI): $m / z$ calcd. for $\mathrm{C}_{20} \mathrm{H}_{22} \mathrm{NO}_{2}{ }^{35} \mathrm{Cl}[\mathrm{M}]^{+}$343.1339; found 343.1340. IR (FT): $v=2921,1635,1460,1383$, $1260,1154,1106 \mathrm{~cm}^{-1}$.

6-Chloro-1,1-diphenyl-2-pivaloylisoindoline (12): $0.37 \mathrm{~g}$ (77\%). Mp 166-168 ${ }^{\circ} \mathrm{C} .{ }^{1} \mathrm{H}$ NMR (500 MHz, $\mathrm{CDCl}_{3}$ ): $\delta=7.24-7.18$ (m, $\left.10 \mathrm{H}, 2 \mathrm{Ph}\right), 7.15$ (s, $\left.1 \mathrm{H}, \mathrm{H}-7\right), 7.10$ (d, $\left.J=8 \mathrm{~Hz}, 1 \mathrm{H}, \mathrm{H}-5\right), 6.85$ (d, $J=8 \mathrm{~Hz}$, $1 \mathrm{H}, \mathrm{H}-4), 5.14$ (s, $2 \mathrm{H}, \mathrm{H}-3), 1.18$ [s, 9H, C( $\left.\left(\mathrm{CH}_{3}\right)_{3}\right] .{ }^{13} \mathrm{C}$ NMR (125 MHz, $\left.\mathrm{CDCl}_{3}\right): \delta=174.4(\mathrm{~s}, \mathrm{C}=\mathrm{O})$, 147.9 (s, C-7a), 142.0 (s, C-1 of 2 Ph), 133.9 (s, C-3a), 133.8 (s, C-6), 128.3 (d, C-3/C-5 of 2 Ph), 128.1 (d, C-4), 127.7 (d, C-2/C-6 of 2 Ph), 126.9 (d, C-4 of 2 Ph), 124.9 (d, C-7), 123.2 (d, C-5), 80.5 (s, C-1), 
53.0 (t, C-3), 39.7 [s, $\left.C\left(\mathrm{CH}_{3}\right)_{3}\right], 27.6$ [q, $\left.\left.\mathrm{C}\left(\mathrm{CH}_{3}\right)_{3}\right] . \mathrm{MS}\left(\mathrm{ES}^{+}\right): \mathrm{m} / \mathrm{z}(\%)=392(33){ }^{37} \mathrm{Cl} \mathrm{MH}\right]^{+}, 390(100)$ $\left[{ }^{35} \mathrm{Cl} \mathrm{MH}\right]^{+}$. HRMS $\left(\mathrm{ES}^{+}\right): \mathrm{m} / z$ calcd. for $\mathrm{C}_{25} \mathrm{H}_{25} \mathrm{NO}^{35} \mathrm{Cl}[\mathrm{MH}]^{+}$390.1625; found 390.1630. IR (FT): $v=$ 2921, 1632, 1420, 1310, 1291, 1113, $1023 \mathrm{~cm}^{-}{ }^{1}$.

6-Fluoro-1-(4-methoxyphenyl)-2-pivaloylisoindoline (13): 0.40 g (84\%). Oil. ${ }^{1} \mathrm{H}$ NMR (500 MHz, $\mathrm{CDCl}_{3}$ ): $\delta=7.20$ (m, $\left.1 \mathrm{H}, \mathrm{H}-4\right), 7.09$ (d, $J=9$ Hz, 2 H, H-2/H-6 of 4-methoxyphenyl) 6.92 (m, $1 \mathrm{H}, \mathrm{H}-5$ ), 6.72 (d, $J=9$ Hz, 2 H, H-3/H-5 of 4-methoxyphenyl), 6.61 (m, 1 H, H-7), 6.21 (s, 1 H, H-1), 5.12-5.07 (m, $2 \mathrm{H}, \mathrm{H}-3 \mathrm{a}$ and $\mathrm{H}-3 \mathrm{~b}), 3.70$ (s, $\left.3 \mathrm{H}, \mathrm{OCH}_{3}\right), 1.23$ [s, $\left.9 \mathrm{H}, \mathrm{C}\left(\mathrm{CH}_{3}\right)_{3}\right] .{ }^{13} \mathrm{C} \mathrm{NMR}\left(125 \mathrm{MHz}, \mathrm{CDCl}_{3}\right): \delta=$ 177.6 (s, C=O), 162.8 (appears as two lines due to coupling with F, C-6), 158.9 (s, C-4 of 4-methoxyphenyl), 142.7 (s, C-7a), 134.5 (s, C-3a), 130.9 (s, C-1 of 4-methoxyphenyl), 127.9 (d, C-2/C-6 of 4-methoxyphenyl), 123.7 (appears as two lines due to coupling with F, C-4), 115.3 (appears as two lines due to coupling with F, C-7), 114.1 (d, C-3/C-5 of 4-methoxyphenyl), 110.6 (appears as two lines due to coupling with F, C-5), 69.0 (d, C-1), 55.2 (q, $\left.\mathrm{CH}_{3}\right), 53.1$ (t, C-3), 39.4 [s, $\left.C\left(\mathrm{CH}_{3}\right)_{3}\right], 27.4$ [q, $\left.\mathrm{C}\left(\mathrm{CH}_{3}\right)_{3}\right] . \mathrm{MS}\left(\mathrm{ES}^{+}\right): \mathrm{m} / \mathrm{z}(\%)=328(100)[\mathrm{MH}]^{+} 146$ (12), 130 (8). HRMS (ES $\left.{ }^{+}\right): \mathrm{m} / \mathrm{z}$ calcd. for $\mathrm{C}_{20} \mathrm{H}_{23} \mathrm{NO}_{2} \mathrm{~F}[\mathrm{MH}]^{+}$328.1713; found 328.1709. IR (FT): $v=2963,1722,1440,1311,1199,1161,1011$ $\mathrm{cm}^{-1}$.

6-Fluoro-1,1-diphenyl-2-pivaloylisoindoline (14): $0.40 \mathrm{~g}$ (84\%). Oil. ${ }^{1} \mathrm{H}$ NMR (500 MHz, $\left.\mathrm{CDCl}_{3}\right): \delta=$ 7.20-7.09 (m, 11 H, 2 Ph and H-7), 6.85 (m, 1 H, H-4), 6.58 (m, 1 H, H-5), 5.18 (s, 2 H, H-3), 1.20 [s, 9 $\left.\mathrm{H}, \mathrm{C}\left(\mathrm{CH}_{3}\right)_{3}\right] .{ }^{13} \mathrm{C} \mathrm{NMR}\left(125 \mathrm{MHz}, \mathrm{CDCl}_{3}\right): \delta=177.1$ (s, C=O), 163.4 (appears as two lines due to coupling with F, C-6), 147.6 (s, C-7a), 141.1 (s, C-1 of 2 Ph), 129.8 (s, C-3a), 128.2 (d, C-3/C-5 of 2 Ph), 127.9 (d, C-2/C-6 of $2 \mathrm{Ph}$ ), 127.3 (s, C-4 of $2 \mathrm{Ph}$ ), 123.3 (appears as two lines due to coupling with $\mathrm{F}$, C-4), 115.6 (appears as two lines due to coupling with F, C-7), 111.7 (appears as two lines due to coupling with F, C-5), 81.5 (d, C-1), 53.2 (t, C-3), 40.1 [s, $C\left(\mathrm{CH}_{3}\right)_{3}$ ], 27.2 [q, $\left.\mathrm{C}\left(\mathrm{CH}_{3}\right)_{3}\right] . \mathrm{MS}\left(\mathrm{ES}^{+}\right): \mathrm{m} / \mathrm{z}$ $(\%)=374(100)[\mathrm{MH}]^{+}, 273$ (6). HRMS $\left(\mathrm{ES}^{+}\right): \mathrm{m} / \mathrm{z}$ calcd. for $\mathrm{C}_{25} \mathrm{H}_{25} \mathrm{NOF}[\mathrm{MH}]^{+}$374.1920; found 374.1914. IR (FT): $v=2931,1612,1496,1454,1411,1289,1072 \mathrm{~cm}^{-1}$.

1-(4-Methoxyphenyl)-2-dimethylaminocarbonylisoindoline (15): 0.42 g (89\%). Mp 111-112 ${ }^{\circ} \mathrm{C} .{ }^{1} \mathrm{H}$ NMR (400 MHz, $\mathrm{CDCl}_{3}$ ): $\delta=$ 7.28-7.21 (m, 5 H, H-4, H-5, H-6 and H-2/H-6 of 4-methoxyphenyl), 7.03 (d, $J=8 \mathrm{~Hz}, 1 \mathrm{H}, \mathrm{H}-7$ ), 6.83 (d, $J=9 \mathrm{~Hz}, 2 \mathrm{H}, \mathrm{H}-3 / \mathrm{H}-5$ of 4-methoxyphenyl), 6.37 (d, $J=3 \mathrm{~Hz}, 1 \mathrm{H}, \mathrm{H}-1$ ), 5.10 (dd, $J=3,14 \mathrm{~Hz}, 1 \mathrm{H}, \mathrm{H}-3 \mathrm{a}$ ), 4.66 (d, $J=14 \mathrm{~Hz}, 1 \mathrm{H}, \mathrm{H}-3 \mathrm{~b}$ ), 3.78 (s, $3 \mathrm{H}, \mathrm{OMe}$ ), 2.90 [s, $\left.6 \mathrm{H}, \mathrm{N}\left(\mathrm{CH}_{3}\right)_{2}\right] .{ }^{13} \mathrm{C}$ NMR (100 MHz, $\mathrm{CDCl}_{3}$ ): $\delta=163.4$ (s, C=O), 159.2 (s, C-4 of 4-methoxyphenyl), 142.2 (s, C-7a), 136.6 (s, C-3a), 136.3 (s, C-1 of 4-methoxyphenyl), 128.8 (d, C-2/C-6 of 4-methoxyphenyl), 128.0 (d, C-4), 127.8 (d, C-7), 123.9 (d, C-6), 122.4 (d, C-5), 114.3 (d, C-3/C-5 of 4-methoxyphenyl), 67.8 (d, C-1), 55.6 (q, OMe), 55.5 (t, C-3), 38.7 [q, N( $\left.\left(\mathrm{CH}_{3}\right)_{2}\right] . \mathrm{MS}(\mathrm{EI}): \mathrm{m} / \mathrm{z}$ (\%) = 296 (7) [M] ${ }^{+}, 252$ (11), 224 (23), 208 (13), 194 
(4), 180 (5), 165 (7), 135 (5), 116 (8), 87 (12), 72 (100), 44 (22). MS (CI): m/z (\%) = 297 (100) [MH] , 91 (14). HRMS (CI): $m / z$ calcd. for $\mathrm{C}_{18} \mathrm{H}_{21} \mathrm{~N}_{2} \mathrm{O}_{2}[\mathrm{MH}]^{+}$297.1598; found 297.1598. IR (FT): $v=2959,1613$, 1510, 1371, 1357, 1243, $1028 \mathrm{~cm}^{-1}$. Anal. Calcd for $\mathrm{C}_{18} \mathrm{H}_{20} \mathrm{~N}_{2} \mathrm{O}_{2}$ (296.152): C 72.95, H 6.80, N 9.45. Found: C 72.80, H 6.78, N 9.30\%.

2-Dimethylaminocarbonyl-1,1-diphenylisoindoline (16): 0.43 g (91\%). Mp 161-163 ${ }^{\circ} \mathrm{C} .{ }^{1} \mathrm{H}$ NMR (400 MHz, $\mathrm{CDCl}_{3}$ ): $\delta=7.29-7.09$ (m, 13 H, 2 Ph, H-4, H-5 and H-6), 6.90 (d, J = 8 Hz, 1 H, H-7), 4.90 (s, 2 H, H-3), 2.61 [s, $\left.6 \mathrm{H}, \mathrm{N}\left(\mathrm{CH}_{3}\right)_{2}\right] .{ }^{13} \mathrm{C}$ NMR (100 MHz, $\left.\mathrm{CDCl}_{3}\right): \delta=160.7$ (s, C=O), 146.1 (s, C-7a), 142.3 (s, C-1 of 2 Ph), 134.7 (s, C-3a), 127.6 (d, C-2/C-6 of 2 Ph), 126.7 (d, C-4), 126.5 (d, C-3/C-5 of 2 Ph), 126.3 (d, C-7), 125.7 (d, C-4 of 2 Ph), 123.4 (d, C-6), 120.9 (d, C-5), 80.0 (s, C-1), 52.5 (t, C-3), 38.0 [q, $\left.\mathrm{N}\left(\mathrm{CH}_{3}\right)_{2}\right] . \mathrm{MS}(\mathrm{EI}): \mathrm{m} / \mathrm{z}(\%)=342$ (10) [M] $]^{+}, 265$ (22) ${ }^{+}, 254$ (24), 239 (9), 193 (7), 178 (15), 165 (20), 72 (100), 42 (10). MS (CI): m/z (\%) = 343 (100) [MH] , 272 (10), 106 (12), 89 (16), 63 (17), 52 (89), 46 (47). HRMS (CI): $m / z$ calcd. for $\mathrm{C}_{23} \mathrm{H}_{23} \mathrm{~N}_{2} \mathrm{O}[\mathrm{MH}]^{+}$343.1805; found 343.1809. IR (FT): $v=2957,1617,1510$, $1371,1358,1242,1173,1037 \mathrm{~cm}^{-1}$.

2-Dimethylaminocarbonyl-6-methoxy-1-(4-methoxyphenyl)isoindoline (17): 0.43 g (91\%). Mp 98-100 ${ }^{\circ}$. ${ }^{1} \mathrm{H}$ NMR (400 MHz, $\mathrm{CDCl}_{3}$ ): $\delta=7.12$ (d, $J=9 \mathrm{~Hz}, 2 \mathrm{H}, \mathrm{H}-2 / \mathrm{H}-6$ of 4-methoxyphenyl), 7.05 (d, $J=8 \mathrm{~Hz}, 1 \mathrm{H}, \mathrm{H}-4), 6.74-6.71$ (m, 3 H, H-5 and H-3/H-5 of 4-methoxyphenyl), 6.42 (d, $J=2 \mathrm{~Hz}, 1 \mathrm{H}, \mathrm{H}-7$ ), 6.23 (d, $J=3$ Hz, 1 H, H-1), 4.90 (dd, $J=3,13$ Hz, 1 H, H-3a), 4.46 (d, $J=13$ Hz, 1 H, H-3b), 3.67 (s, 3 H, $\left.\mathrm{OCH}_{3}\right), 3.61$ (s, $\left.3 \mathrm{H}, \mathrm{OCH}_{3}\right), 2.77$ [s, $\left.6 \mathrm{H}, \mathrm{N}\left(\mathrm{CH}_{3}\right)_{2}\right] .{ }^{13} \mathrm{C} \mathrm{NMR}\left(100 \mathrm{MHz}, \mathrm{CDCl}_{3}\right): \delta=163.4(\mathrm{~s}, \mathrm{C}=\mathrm{O})$, 160.0 (s, C-6), 159.3 (s, C-4 of 4-methoxyphenyl), 143.6 (s, C-7a), 136.2 (s, C-1 of 4-methoxyphenyl), 129.7 (d, C-2/C-6 of 4-methoxyphenyl), 129.1 (s, C-3a), 123.5 (d, C-4), 115.0 (d, C-7), 114.7 (d, C-3/C-5 of 4-methoxyphenyl), 108.9 (d, C-5), 67.9 (d, C-1), 55.8 (q, $\mathrm{OCH}_{3}$ ), 55.7 (q, OCH $\mathrm{OCH}_{3}, 55.0$ (t, C-3), 38.7 [q, $\left.\mathrm{N}\left(\mathrm{CH}_{3}\right)_{2}\right]$. MS (EI): m/z (\%) = 326 (3) [M] , 282 (5), 254 (10), 238 (16), 218 (5), 146 (4), 72 (100), 44 (10). MS (CI): $m / z(\%)=327(100)[\mathrm{MH}]^{+}, 105$ (5), 91 (6), 52 (8), 46 (13). HRMS (CI): m/z calcd. for $\mathrm{C}_{19} \mathrm{H}_{23} \mathrm{~N}_{2} \mathrm{O}_{3}[\mathrm{MH}]^{+}$327.1703; found 327.1702. IR (FT): $v=2931,1616,1511,1400,1370,1251,1023$ $\mathrm{cm}^{-1}$. Anal. Calcd for $\mathrm{C}_{19} \mathrm{H}_{22} \mathrm{~N}_{2} \mathrm{O}_{3}$ (326.163): C 69.92, H 6.79, N 8.58. Found: C 69.97, H 6.73, N 5.42\%.

2-Dimethylaminocarbonyl-1,1-diphenyl-6-methoxyisoindoline (18): 0.42 g (88\%). Mp 157-158 ${ }^{\circ} \mathrm{C} .{ }^{1} \mathrm{H}$ NMR (400 MHz, $\mathrm{CDCl}_{3}$ ): $\delta=7.29-7.11$ (m, 10 H, 2 Ph), 7.08 (d, $J=8$ Hz, 1 H, H-4), 6.70 (dd, $J=2,8$ Hz, $1 \mathrm{H}, \mathrm{H}-5), 6.42$ (d, $J=2 \mathrm{~Hz}, 1 \mathrm{H}, \mathrm{H}-7), 4.85$ (s, $2 \mathrm{H}, \mathrm{H}-3$ ), 3.58 (s, $\left.\mathrm{OCH}_{3}\right), 2.61$ [s, $\left.6 \mathrm{H}, \mathrm{N}\left(\mathrm{CH}_{3}\right)_{2}\right] .{ }^{13} \mathrm{C}$ NMR (100 MHz, $\mathrm{CDCl}_{3}$ ): $\delta=162.0$ (s, C=O), 159.9 (s, C-6), 148.9 (s, C-7a), 143.6 (s, C-1 of 2 Ph), 129.3 (d, C-3/C-5 of 2 Ph), 128.2 (s, C-3a), 128.0 (d, C-2/C-6 of 2 Ph), 127.2 (d, C-4 of 2 Ph), 123.1 (d, C-4), 114.3 (d, C-5), 109.9 (d, C-7), 79.5 (s, C-1), 55.8 (q, $\left.\mathrm{OCH}_{3}\right), 53.6$ (t, C-3), 39.5 [q, N(CH3) $]$. MS (EI): m/z (\%) = 372 (20) [M]+, 295 (6), 284 (41), 165 (7), 91 (8), 88 (19), 72 (100), 42 (22). MS (CI): m/z (\%) = 373 (100) 
$[\mathrm{MH}]^{+}$. HRMS (CI): $\mathrm{m} / \mathrm{z}$ calcd. for $\mathrm{C}_{24} \mathrm{H}_{25} \mathrm{~N}_{2} \mathrm{O}_{2}[\mathrm{MH}]^{+}$373.1911; found 373.1911. IR (FT): $v=2997,1617$, 1510, 1371, 1242, 1172, $1029 \mathrm{~cm}^{-1}$. Anal. Calcd for $\mathrm{C}_{24} \mathrm{H}_{24} \mathrm{~N}_{2} \mathrm{O}_{2}$ (372.184): C 77.39, H 6.49, N 7.52. Found: C 77.37, H 6.51, N 7.47\%.

2-Dimethylaminocarbonyl-1-(4-methoxyphenyl)-6-methylisoindoline (19): 0.43 g (91\%). Mp 125-126 ${ }^{\circ} \mathrm{C} .{ }^{1} \mathrm{H}$ NMR (400 MHz, $\mathrm{CDCl}_{3}$ ): $\delta=7.13$ (d, $J=9 \mathrm{~Hz}, 2 \mathrm{H}, \mathrm{H}-2 / \mathrm{H}-6$ of 4-methoxyphenyl), 7.05 (d, $J=8$ Hz, 1 H, H-4), 6.97 (d, $J=8$ Hz, 1 H, H-5), 6.75-6.73 (m, 3 H, H-7 and H-3/H-5 of 4-methoxyphenyl), 6.22 (d, $J=2 \mathrm{~Hz}, 1 \mathrm{H}, \mathrm{H}-1$ ), 4.94 (dd, $J=2,14 \mathrm{~Hz}, 1 \mathrm{H}, \mathrm{H}-3 \mathrm{a}$ ), 4.50 (d, $J=14 \mathrm{~Hz}, 1 \mathrm{H}, \mathrm{H}-3 \mathrm{~b}$ ), 3.68 (s, 3 H, $\left.\mathrm{OCH}_{3}\right), 2.78$ [s, $6 \mathrm{H}, \mathrm{N}\left(\mathrm{CH}_{3}\right)_{2}$ ], 2.18 (s, $\left.3 \mathrm{H}, \mathrm{CH}_{3}\right) .{ }^{13} \mathrm{C} \mathrm{NMR}\left(100 \mathrm{MHz}, \mathrm{CDCl}_{3}\right): \delta=163.4$ (s, C=O), 159.2 (s, C-4 of 4-methoxyphenyl), 142.3 (s, C-7a), 137.7 (s, C-6), 136.4 (s, C-1 of 4-methoxyphenyl), 133.6 (s, C-3a), 128.5 (d, C-2/C-6 of 4-methoxyphenyl), 128.2 (d, C-7), 124.3 (d, C-4), 122.2 (d, C-5), 114.3 (d, C-3/C-5 of 4-methoxyphenyl), 67.8 (d, C-1), 55.6 (q, $\left.\mathrm{OCH}_{3}\right), 55.3$ (t, C-3), 38.7 [q, N( $\left.\mathrm{CH}_{3}\right)_{2}$ ], 15.7 (q, $\mathrm{CH}_{3}$ ). MS (EI): m/z (\%) = 310 (5) [M] , 266 (14), 238 (22), 222 (19), 165 (13), 130 (12), 72 (100), 44 (15), 42 (23). MS (CI): $m / z(\%)=311(100)[\mathrm{MH}]^{+}, 52$ (22). HRMS (CI): $m / z$ calcd. for $\mathrm{C}_{19} \mathrm{H}_{23} \mathrm{~N}_{2} \mathrm{O}_{2}[\mathrm{MH}]^{+}$ 311.1754; found 311.1749. IR (FT): $v=2958,1616,1511,1371,1358,1243,1029 \mathrm{~cm}^{-1}$. Anal. Calcd for $\mathrm{C}_{19} \mathrm{H}_{22} \mathrm{~N}_{2} \mathrm{O}_{2}$ (310.168): C 73.52, H 7.14, N 9.03. Found: C 73.50, H 7.15, N 9.01\%.

2-Dimethylaminocarbonyl-1,1-diphenyl-6-methylisoindoline (20): 0.42 g (88\%). Mp $158-160{ }^{\circ} \mathrm{C} .{ }^{1} \mathrm{H}$ NMR (400 MHz, $\mathrm{CDCl}_{3}$ ): $\delta=7.27-7.13$ (m, 10 H, 2 Ph), 7.07 (d, $J=8$ Hz, 1 H, H-4), 6.95 (d, $J=8$ Hz, 1 H, H-5), 6.72 (s, 1 H, H-7), 3.25 (s, 2 H, H-3), 2.61 [s, $6 \mathrm{H}, \mathrm{N}\left(\mathrm{CH}_{3}\right)_{2}$ ], 2.15 (s, $\left.3 \mathrm{H}, \mathrm{CH}_{3}\right) .{ }^{13} \mathrm{C}$ NMR (100 MHz, $\mathrm{CDCl}_{3}$ ): $\delta=161.9$ (s, C=O), 147.6 (s, C-7a), 143.8 (s, C-1 of 2 Ph), 137.9 (s, C-6), 133.1 (s, C-3a), 129.0 (d, C-3/C-5 of 2 Ph), 128.8 (d, C-7), 127.9 (d, C-2/C-6 of 2 Ph), 127.1 (d, C-4 of 2 Ph), 125.2 (d, C-4), 122.1 (d, C-5), 79.4 (s, C-1), 53.8 (t, C-3), 39.5 [q, N( $\left.\mathrm{CH}_{3}\right)_{2}$ ], 21.9 (q, $\left.\mathrm{CH}_{3}\right) . \mathrm{MS}(\mathrm{EI}): \mathrm{m} / \mathrm{z}(\%)=356$ (7) [M] $]^{+}, 279$ (13), 268 (31), 253 (7), 165 (13), 72 (100), 42 (15). MS (CI): m/z (\%) = 357 (100) [MH], 193 (12), 106 (14), 89 (16), 46 (30). HRMS (CI): m/z calcd. for $\mathrm{C}_{24} \mathrm{H}_{25} \mathrm{~N}_{2} \mathrm{O}$ [MH] 357.1961; found 357.1960. IR (FT): $v=2852,1619,1520,1363,1340,1218 \mathrm{~cm}^{-1}$.

6-Chloro-2-dimethylaminocarbonyl-1-(4-methoxyphenyl)isoindoline (21): 0.37 g (78\%). Oil. ${ }^{1} \mathrm{H}$ NMR (500 MHz, $\mathrm{CDCl}_{3}$ ): $\delta=7.25$ (s, $1 \mathrm{H}, \mathrm{H}-7$ ), 7.20 (d, $J=8 \mathrm{~Hz}, 1 \mathrm{H}, \mathrm{H}-5$ ), 7.10 (d, $J=9 \mathrm{~Hz}, 2 \mathrm{H}$, H-2/H-6 of 4-methoxyphenyl), 6.90 (d, $J=8 \mathrm{~Hz}, 1 \mathrm{H}, \mathrm{H}-4), 6.75$ (d, $J=9 \mathrm{~Hz}, 2 \mathrm{H}, \mathrm{H}-3 / \mathrm{H}-5$ of 4-methoxyphenyl), 6.25 (d, $J=3 \mathrm{~Hz}, 1 \mathrm{H}, \mathrm{H}-1$ ), 4.95 (dd, $J=3,14 \mathrm{~Hz}, 1 \mathrm{H}, \mathrm{H}-3 \mathrm{a}$ ), 4.50 (d, $J=14 \mathrm{~Hz}, 1$ $\mathrm{H}, \mathrm{H}-3 \mathrm{~b}), 3.65$ (s, $\left.3 \mathrm{H}, \mathrm{OCH}_{3}\right), 2.75$ [s, $\left.6 \mathrm{H}, \mathrm{N}\left(\mathrm{CH}_{3}\right)_{2}\right] .{ }^{13} \mathrm{C} \mathrm{NMR}\left(125 \mathrm{MHz}, \mathrm{CDCl}_{3}\right): \delta=162.8(\mathrm{~s}, \mathrm{C}=\mathrm{O})$, 159.1 (s, C-4 of 4-methoxyphenyl), 143.8 (s, C-7a), 135.1 (s, C-3a), 134.7 (s, C-6), 133.4 (s, C-1 of 4-methoxyphenyl) 128.3 (d, C-2/C-6 of 4-methoxyphenyl), 127.8 (d, C-4), 123.8 (d, C-7), 123.3 (d, C-5), 114.0 (d, C-3/C-5 of 4-methoxyphenyl), 67.2 (d, C-1), 55.2 (q, $\left.\mathrm{OCH}_{3}\right), 54.6$ (t, C-3), 38.3 [q, N( $\left.\mathrm{CH}_{3}\right)_{2}$ ]. 
MS (APCI): $m / z(\%)=333(33)\left[{ }^{37} \mathrm{Cl} \mathrm{MH}\right]^{+}, 331(100)\left[{ }^{35} \mathrm{Cl} \mathrm{MH}\right]^{+}, 243$ (10), 223 (22). HRMS (APCI): $\mathrm{m} / \mathrm{z}$ calcd. for $\mathrm{C}_{18} \mathrm{H}_{20} \mathrm{~N}_{2} \mathrm{O}_{2}{ }^{35} \mathrm{Cl}[\mathrm{MH}]^{+}$331.1213; found 331.1215. IR (FT): $v=2963,1644,1485,1438$, $1261,1098 \mathrm{~cm}^{-1}$.

6-Chloro-2-dimethylaminocarbonyl-1-phenylisoindoline (22): 0.37 g (78\%). Oil. ${ }^{1} \mathrm{H}$ NMR (500 MHz, $\mathrm{CDCl}_{3}$ ): $\delta=7.25$ (s, $\left.1 \mathrm{H}, \mathrm{H}-7\right), 7.20-7.10$ (m, $5 \mathrm{H}, \mathrm{Ph}$ ), 7.09 (d, $\left.J=8 \mathrm{~Hz}, 1 \mathrm{H}, \mathrm{H}-5\right), 6.90$ (d, J = 8 Hz, 1 H, H-4), 6.25 (d, $J=3$ Hz, 1 H, H-1), 4.95 (dd, $J=3,14$ Hz, 1 H, H-3a), 4.53 (d, $J=14$ Hz, 1 H, H-3b), 2.80 [s, $\left.6 \mathrm{H}, \mathrm{N}\left(\mathrm{CH}_{3}\right)_{2}\right] .{ }^{13} \mathrm{C} \mathrm{NMR}\left(125 \mathrm{MHz}, \mathrm{CDCl}_{3}\right): \delta=162.8$ (s, C=O), 143.5 (s, C-7a), 142.8 (s, C-3a), 134.6 (s, C-1 of Ph), 133.5 (s, C-6), 128.7 (d, C-3/C-5 of Ph), 127.9 (d, C-4), 127.7 (d, C-7), 127.0 (d, C-2/C-6 of Ph), 123.7 (d, C-5), 123.4 (d, C-4 of Ph), 67.8 (d, C-1), 54.9 (t, C-3), 38.3 [q, N( $\left.\mathrm{CH}_{3}\right)_{2}$ ]. MS (APCI): $m / z(\%)=303$ (33) $\left[{ }^{37} \mathrm{Cl} \mathrm{MH}\right]^{+}, 301$ (100) $\left[{ }^{35} \mathrm{Cl} \mathrm{MH}\right]^{+}, 256$ (10), 213 (13). HRMS (APCI): $m / z$ calcd. for $\mathrm{C}_{17} \mathrm{H}_{18} \mathrm{~N}_{2} \mathrm{O}^{35} \mathrm{Cl}[\mathrm{MH}]^{+}$301.1108; found 301.1096. IR (FT): $v=2971,1698,1540,1466,1427$, $1368,1156,1087 \mathrm{~cm}^{-1}$.

2-Dimethylaminocarbonyl-6-fluoro-1-(4-methoxyphenyl)isoindoline (23): $0.40 \mathrm{~g}$ (85\%). Oil. ${ }^{1} \mathrm{H}$ NMR (500 MHz, $\mathrm{CDCl}_{3}$ ): $\delta=7.15$ (d, $J=9 \mathrm{~Hz}, 2 \mathrm{H}, \mathrm{H}-2 / \mathrm{H}-6$ of 4-methoxyphenyl), 7.10 (m, $1 \mathrm{H}, \mathrm{H}-4$ ), 6.90 (m, $1 \mathrm{H}, \mathrm{H}-5$ ), 6.75 (d, $J=9$ Hz, 2 H, H-3/H-5 of 4-methoxyphenyl), 6.60 (m, $1 \mathrm{H}, \mathrm{H}-7$ ), 6.28 (s, $1 \mathrm{H}$, H-1), 4.92 (d, $J=13 \mathrm{~Hz}, 1 \mathrm{H}, \mathrm{H}-3 \mathrm{a}), 4.50$ (d, $J=13 \mathrm{~Hz}, 1 \mathrm{H}, \mathrm{H}-3 \mathrm{~b}$ ), 3.70 (s, $3 \mathrm{H}, \mathrm{OCH}_{3}$ ), 2.80 [s, $6 \mathrm{H}$, $\left.\mathrm{N}\left(\mathrm{CH}_{3}\right)_{2}\right] .{ }^{13} \mathrm{C} \mathrm{NMR}\left(125 \mathrm{MHz}, \mathrm{CDCl}_{3}\right.$ ): $\delta=163.7$ (s, C=O), 162.3 (appears as two lines due to coupling with F, C-6), 159.1 (s, C-4 of 4-methoxyphenyl), 143.9 (s, C-7a), 135.2 (s, C-3a), 131.6 (s, C-1 of 4-methoxyphenyl), 128.3 (d, C-2/C-6 of 4-methoxyphenyl), 123.4 (appears as two lines due to coupling with F, C-4), 114.9 (appears as two lines due to coupling with F, C-7), 114.0 (d, C-3/C-5 of 4-methoxyphenyl), 110.5 (appears as two lines due to coupling with F, C-5), 67.4 (d, C-1), 55.2 (q, $\mathrm{CH}_{3}$ ), 54.5 (t, C-3), 38.3 [q, N(CH$)_{2}$ ]. MS (ES $\left.{ }^{+}\right): m / z(\%)=315$ (100) [MH], ${ }^{+} 146$ (15), 130 (8). HRMS (ES $\left.{ }^{+}\right):$ $\mathrm{m} / \mathrm{z}$ calcd. for $\mathrm{C}_{18} \mathrm{H}_{20} \mathrm{~N}_{2} \mathrm{O}_{2} \mathrm{~F}[\mathrm{MH}]^{+}$315.1509; found 315.1501. IR (FT): $v=2960,1639,1465,1423$, $1367,1321,1251,1133,1072 \mathrm{~cm}^{-1}$.

2-Dimethylaminocarbonyl-6-fluoro-1-phenylisoindoline (24): 0.40 g (85\%). Oil. ${ }^{1} \mathrm{H}$ NMR (500 MHz, $\mathrm{CDCl}_{3}$ ): $\delta=7.25-7.10$ (m, 6 H, Ph, H-7), 6.85 (m, 1 H, H-4), 6.63 (m, 1 H, H-5), 6.30 (s, 1 H, H-1), 5.00 (d, $J=13 \mathrm{~Hz}, 1 \mathrm{H}, \mathrm{H}-3 \mathrm{a}), 4.55$ (d, $J=13 \mathrm{~Hz}, 1 \mathrm{H}, \mathrm{H}-3 \mathrm{~b}), 2.82$ [s, $\left.6 \mathrm{H}, \mathrm{N}\left(\mathrm{CH}_{3}\right)_{2}\right] .{ }^{13} \mathrm{C} \mathrm{NMR}(125 \mathrm{MHz}$, $\mathrm{CDCl}_{3}$ ): $\delta=163.7$ (s, C=O), 162.5 (appears as two lines due to coupling with F, C-6), 143.2 (s, C-7a), 142.4 (s, C-1 of Ph), 131.2 (s, C-3a), 129.0 (d, C-3/C-5 of Ph), 127.9 (d, C-4 of Ph), 126.9 (d, C-2/C-6 of $\mathrm{Ph}$ ), 123.5 (appears as two lines due to coupling with F, C-4), 115.2 (appears as two lines due to coupling with F, C-7), 110.6 (appears as two lines due to coupling with F, C-5), 68.2 (d, C-1), 54.8 (t, C-3), 38.5 
[q, $\left.\mathrm{N}\left(\mathrm{CH}_{3}\right)_{2}\right] . \mathrm{MS}\left(\mathrm{ES}^{+}\right): \mathrm{m} / \mathrm{z}(\%)=285(100)[\mathrm{MH}]^{+}, 197$ (8). HRMS (ES $\left.{ }^{+}\right): m / z$ calcd. for $\mathrm{C}_{17} \mathrm{H}_{18} \mathrm{~N}_{2} \mathrm{OF}$ $[\mathrm{MH}]^{+}$285.1403; found 285.1392. IR (FT): $v=2951,1632,1586,1517,1486,1376,1285,1178,1038$ $\mathrm{cm}^{-1}$.

$N$-(2-(Cyclohexenyl)pivalamides 27 and 28: The procedure was identical with that described for the synthesis of 5 except that compounds 25 and 26 (0.50 g) were used as starting materials instead of $\mathbf{4}$. The reaction mixtures were worked-up and the residues obtained were subjected to flash column chromatography (silica gel; $\mathrm{Et}_{2} \mathrm{O}$-hexane, 1:3) to give the pure products $\mathbf{2 7}$ and $\mathbf{2 8}$ as white solids.

$\mathrm{N}$-(2-Cyclohexen-1-ylbenzyl)pivalamide (27): 0.38 g (82\%). Mp 118-119 ${ }^{\circ} \mathrm{C} .{ }^{1} \mathrm{H}$ NMR (400 MHz, $\mathrm{CDCl}_{3}$ ): $\delta=7.19-7.03$ (m, 4 H, H-3, H-4, H-5 and H-6), 5.78 (br, exch., 1 H, NH), 5.52 (m, 1 H, H-2 of cyclohexenyl), 4.34 (d, $J=5$ Hz, 2 H, CH 2 ), 2.15-2.07 (m, 4 H, H-3 and H-6 of cyclohexenyl), 1.71-1.58 (m, $4 \mathrm{H}, \mathrm{H}-4$ and $\mathrm{H}-5$ of cyclohexenyl), 1.13 [s, $\left.9 \mathrm{H}, \mathrm{C}\left(\mathrm{CH}_{3}\right)_{3}\right] .{ }^{13} \mathrm{C} \mathrm{NMR}\left(100 \mathrm{MHz}, \mathrm{CDCl}_{3}\right): \delta=178.4(\mathrm{~s}$, C=O), 144.8 (s, C-1 of cyclohexenyl), 138.4 (s, C-1), 135.5 (s, C-2), 129.3 (d, C-5), 128.9 (d, C-6), 127.7 (d, C-3), 127.4 (d, C-4), 127.2 (d, C-2 of cyclohexenyl), 42.2 (t, $\left.\mathrm{CH}_{2} \mathrm{NH}\right), 39.1$ [s, $C\left(\mathrm{CH}_{3}\right)_{3}$ ], 31.0 (t, C-6 of cyclohexenyl), 28.0 [q, C( $\left.\mathrm{CH}_{3}\right)_{3}$ ], 25.8 (t, C-3 of cyclohexenyl), 23.4 (t, C-5 of cyclohexenyl), 22.4 (t, C-4 of cyclohexenyl). MS (EI): m/z (\%) = 271 (4) [M] $]^{+}, 186$ (8), 171 (17), 170 (56), 157 (11), 142 (50), 128 (32), 115 (26), 102 (12), 91 (11), 77 (5), 57 (100), 41 (71). MS (CI): m/z (\%) = 543 (4) [2 M + 1] $]^{+}, 289$ (6) $\left[\mathrm{M}+\mathrm{NH}_{4}\right]^{+}, 272$ (100) [MH] $]^{+}, 170$ (15), 119 (17), 102 (9), 52 (24). HRMS (CI): m/z calcd. for $\mathrm{C}_{18} \mathrm{H}_{26} \mathrm{NO}$ $[\mathrm{MH}]^{+}$272.2009; found 272.2012. IR (FT): $v=3328,2921,1638,1512,1540,1368,1210,1003 \mathrm{~cm}^{-1}$. Anal. Calcd for $\mathrm{C}_{18} \mathrm{H}_{25} \mathrm{NO}$ (271.194): C 79.66, H 9.28, N 5.16. Found: C 79.73, H 9.33, N 5.09\%.

$N$-(2-Cyclohexen-1-yl-4-methoxybenzyl)pivalamide (28): 0.39 g (83\%). Mp 99-100 ${ }^{\circ} \mathrm{C} .{ }^{1} \mathrm{H}$ NMR (400 $\mathrm{MHz}, \mathrm{CDCl}_{3}$ ): $\delta=7.09$ (d, $J=8 \mathrm{~Hz}, 1 \mathrm{H}, \mathrm{H}-6$ ), 6.68 (dd, $J=2,8 \mathrm{~Hz}, 1 \mathrm{H}, \mathrm{H}-5$ ), 6.58 (d, $J=2 \mathrm{~Hz}, 1 \mathrm{H}, \mathrm{H}-3$ ), 5.70 (br, exch., 1 H, NH), 5.52 (m, 1 H, H-2 of cyclohexenyl), 4.26 (d, J = 5 Hz, 2 H, CH 2 ), 3.71 (s, 3 H, $\mathrm{OCH}_{3}$ ), 2.15-2.06 (m, $4 \mathrm{H}, \mathrm{H}-3$ and H-6 of cyclohexenyl), 1.71-1.58 (m, $4 \mathrm{H}, \mathrm{H}-4$ and $\mathrm{H}-5$ of cyclohexenyl), 1.11 [s, $\left.9 \mathrm{H}, \mathrm{C}\left(\mathrm{CH}_{3}\right)_{3}\right] .{ }^{13} \mathrm{C} \mathrm{NMR}\left(100 \mathrm{MHz}, \mathrm{CDCl}_{3}\right): \delta=178.2$ (s, C=O), 159.1 (s, C-4), 146.2 (s, C-1 of cyclohexenyl), 138.5 (s, C-2), 130.5 (d, C-6), 127.7 (s, C-1), 127.1 (d, C-2 of cyclohexenyl), 114.7 (d, C-5), 112.7 (d, C-3), 55.7 (q, $\mathrm{OCH}_{3}$ ), 41.8 (t, $\left.\mathrm{CH}_{2} \mathrm{NH}\right), 39.0$ [s, C( $\left.\mathrm{CH}_{3}\right)_{3}$ ], 30.9 (t, C-6 of cyclohexenyl), 28.0 [q, C $\left(\mathrm{CH}_{3}\right)_{3}$ ], 25.7 (t, C-3 of cyclohexenyl), 23.4 (t, C-5 of cyclohexenyl), 21.9 (t, C-4 of cyclohexenyl). MS (EI): m/z (\%) = 301 (2) [M] $]^{+}, 200$ (22), 172 (13), 159 (9), 128 (6), 115 (8), 77 (5), 57 (100), 41 (93). MS (CI): m/z (\%) = 319 (3) $\left[\mathrm{M}+\mathrm{NH}_{4}\right]^{+}, 302$ (100) [MH] $]^{+}, 200$ (11), 119 (17), 102 (12), 52 (15). HRMS (CI): $m / z$ calcd. for $\mathrm{C}_{19} \mathrm{H}_{28} \mathrm{NO}_{2}[\mathrm{MH}]^{+}$302.2115; found 302.2115. IR (FT): $v=3347$, 2940, 1647, 1505, 1492, 1330, 1207, $1029 \mathrm{~cm}^{-1}$. 


\section{ACKNOWLEDGEMENTS}

We thank Dr Benson Kariuki, X-ray crystallography service at Cardiff School of Chemistry, for the crystal structures and Cardiff University and the Egyptian Government for financial support.

\section{REFERENCES}

1. N. J. Lawrence, J. Liddle, S. M. Bushell, and D. A. Jackson, J. Org. Chem., 2002, 67, 457.

2. A. Couture, E. Deniau, P. Grandclaudon, C. Hoarau, and V. Rys, Tetrahedron Lett., 2002, 43, 2207.

3. J. R. Fuchs and R. L. Funk, Org. Lett., 2001, 3, 3923.

4. J. L. Wood, B. M. Stoltz, and S. N. Goodman, J. Am. Chem. Soc., 1996, 118, 10656.

5. F. G. Fang and S. J. Danishefsky, Tetrahedron Lett., 1989, 30, 2747.

6. E. Valencia, A. J. Freyer, M. Shamma, and V. Fajardo, Tetrahedron Lett., 1984, 25, 599.

7. V. Fajardo, V. Elango, B. K. Cassels, and M. Shamma, Tetrahedron Lett., 1982, 23, 39.

8. G. Stajer and F. Csende, Curr. Org. Chem., 2005, 9, 1277 and references therein.

9. G. E. Marinicheva and T. I. Gubina, Chemistry of Heterocyclic Compounds, 2004, 40, 1517 and references therein.

10. P. V. Reddy and S. V. Bhat, Tetrahedron Lett., 1997, 38, 9039.

11. Y. Watanabe, S. C. Shim, H. Uchida, T. Mitsudo, and Y. Takegami, Tetrahedron, 1979, 35, 1433.

12. S. S. Simons and D. F. Johnson, J. Org. Chem., 1978, 43, 2886.

13. M. J. Haddadin and N. C. Chelhot, Tetrahedron Lett., 1973, 14, 5185.

14. J. C. Emmett and W. Lwowski, Tetrahedron, 1966, 22, 1011.

15. J. Clayden and C. J. Menet, Tetrahedron Lett., 2003, 44, 3059.

16. J. Clayden, C. J. Menet, and D. J. Mansfield, Org. Lett., 2000, 2, 4229.

17. J. Clayden and K. Tchabanenko, Chem. Commun., 2000, 317.

18. A. Ahmed, J. Clayden, and S. A. Yasin, Chem. Commun., 1999, 231.

19. J. Clayden, F. E. Knowles, and I. R. Baldwin, J. Am. Chem. Soc., 2005, 127, 2412.

20. See for example: K. Smith, G. A. El-Hiti, M. A. Abdo, and M. F. Abdel-Megeed, J. Chem. Soc., Perkin Trans. 1, 1995, 1029; K. Smith, G. A. El-Hiti, M. F. Abdel-Megeed, and M. A. Abdo, J. Org. Chem., 1996, 61, 647; K. Smith, G. A. El-Hiti, M. F. Abdel-Megeed, and M. A. Abdo, J. Org. Chem., 1996, 61, 656; K. Smith, G. A. El-Hiti, G. J. Pritchard, and A. Hamilton, J. Chem. Soc., Perkin Trans. 1, 1999, 2299; K. Smith, G. A. El-Hiti, and A. P. Shukla, J. Chem. Soc., Perkin Trans. 1, 1999, 2305; K. Smith, G. A. El-Hiti, and A. C. Hawes, Synthesis, 2003, 2047; K. Smith, G. A. El-Hiti, and S. A. Mahgoub, Synthesis, 2003, 2345; G. A. El-Hiti, Synthesis, 2003, 2799; K. Smith, G. A. El-Hiti, and M. F. Abdel-Megeed, Synthesis, 2004, 2121; G. A. El-Hiti, Synthesis, 2004, 363; K. Smith, G. A. El-Hiti, 
and A. S. Hegazy, J. Sulfur Chem., 2005, 26, 121; K. Smith, G. A. El-Hiti, and A. S. Hegazy, Synthesis, 2005, 2951; K. Smith and M. L. Barratt, J. Org. Chem., 2007, 72, 1031; K. Smith, G. A. El-Hiti, and A. S. Hegazy, Synlett, 2009, 2242.

21. K. Smith, G. A. El-Hiti, A. S. Hegazy, A. Fekri, and B. M. Kariuki, submitted.

22. S. C. Watson and J. F. Eastham, J. Organomet. Chem., 1967, 9, 165.

23. 'Vogel’s Textbook of Practical Organic Chemistry', 5th ed., Longman, Harlow, 1989.

24. D. D. Perrin, W. L. F. Armarego, 'Purification of Laboratory Chemicals', 3rd ed., Pergamon, Oxford, 1988. 